\title{
PEQUENOS PROJETOS EM INTERNET DAS COISAS (IoT) SÃO FERRAMENTAS DE INCENTIVO À PESQUISA E INICIAÇÃO CIENTÍFICA
}

Ana Beatriz Catarim Trizólio-e-mail: abc.trizolio@gmail.com

Evandro Martins Gregoleto - e-mail: evandromartinsgregoleto@gmail.com

Suely Cunha Amaro Mantovani -e-mail: suely.mantovani@unesp.br

Universidade Estadual Paulista, Júlio de Mesquita Filho, Faculdade de Engenharia Elétrica. Endereço - Av. Brasil -Centro n.56

CEP - 15385000 - Ilha Solteira - São Paulo.

Resumo: A internet se consolida cada vez mais no cotidiano das pessoas como ferramenta imprescindivel por apresentar novas tecnologias e interações, principalmente em épocas de pandemia. A Internet das Coisas (IoT), conceito originário da década de 90, que conecta os dispositivos por meio da internet com o homem. É uma linha de pesquisa em pleno desenvolvimento, por isso é crescente o interesse dos alunos da graduação e o desejo de realizar uma iniciação científica sobre o tema. Neste artigo relatam-se dois trabalhos de Iniciação Científica desenvolvidos em Internet das Coisas, sendo que o primeiro utiliza a assistente de voz do smartphone, Google Assistant, para a automação de tarefas como, acender/apagar uma lâmpada, para isso são usados aplicativos na nuvem chamado IFTTT (If This Than That) que faz a conexão entre o serviço da Google Assistant e o Blynk controlando um Arduino Mega 2560, com shield ethernet e relé para acionamento da lâmpada, completando um circuito básico de automação e controle via IoT por comando de voz. No segundo trabalho aborda-se a Internet das Coisas para tornar um ambiente residencial inteligente usando o Arduino UNO R3 para a aquisição/atuação da varáveis envolvidas, sensores de temperatura, umidade, monóxido de carbono e luminosidade. Para o controle do ambiente, monitora-se a leitura das variáveis por meio da conexão wifi do NodeMCU com o servidor do website ThingSpeak.

Palavras-chave: Internet das coisas, Sistemas embarcados, Sensores, Automação de tarefas.

\section{INTRODUÇÃ̃}

Internet das coisas (do inglês, Internet of Things - IoT) refere-se à interconexão digital entre vários dispositivos físicos do cotidiano à rede de internet que se comunicam, trocando informações para diversas finalidades. Isso gerou novos serviços que buscam analisar dados do usuário e proporcionar, mais conforto, segurança e agilidade nas ações.

O avanço de áreas de microeletrônica, telecomunicações e sensoriamento, possibilitou buscar a otimização dos recursos energéticos da natureza, por meio da análise e interpretação de dados do ambiente, da rotina e das ações das pessoas. Utilizando a internet para fazer essa comunicação entre os sensores que captam os dados do mundo físico, e respondem por meio de seus atuadores a esta análise de dados, foi possível revolucionar as diversas áreas como a 
agricultura, transporte, negócios, medicina e acadêmica (LIMA; NOBRE; ALENCAR, 2016; OLIVEIRA, 2017; ROCHA, 2019; SOARES et al, 2019).

$\mathrm{O}$ acesso à internet ampliado e tantos dispositivos conectados possibilitaram muitas outras funcionalidades de comunicação, monitoramento e controle que foram incorporados ao meio doméstico, empresarial e industrial, revolucionando o modo em que vivemos. A evolução da Internet das Coisas tem causado um grande impacto no dia a dia das pessoas. Por meio do avanço desta tecnologia é possível deixar a casa inteligente, com objetos e eletrônicos funcionais e autossuficientes. Ou seja, além dos smartphones e computadores pessoais, outros dispositivos poderão identificar padrões, processar informações e executar tarefas com apenas um (ou nenhum) clique.

Atualmente, são encontrados muitos equipamentos e soluções de automação residencial, conectados e integrados a aplicativos, mas a tecnologia está convergindo para produtos que qualquer pessoa possa instalar, necessitando de pouca infraestrutura para usufruir dos benefícios de uma casa inteligente. Para isso, é necessário apenas ter uma casa preparada para a IoT, com uma excelente conexão à internet, melhorando a qualidade e a segurança de vida das pessoas.

Considera-se que a chamada Indústria 4.0 ou quarta revolução industrial é devido à internet das coisas. Nas "fábricas inteligentes" vários sistemas passaram a ser distribuídos, com processamento no local. Os sensores enviam, por meio da internet, os dados para um software remoto ou para a nuvem, modernizando com este procedimento as empresas e a sua administração.

Trabalhos encontrados na literatura, utilizam os conceitos de IoT, como o de Sousa (2018) que usa o comando de voz visando a automação residencial para pessoas com mobilidade reduzida, ou em Alexandre, Silva, Silva (2019) que aplica IoT a um sistema de irrigação. Por meio da voz do usuário podem ser controladas as entradas/saídas de dados, e sinais PWM possibilitando utilizar o smartphone ou os alto-falantes inteligentes disponíveis no mercado. Este processo pode ser aplicado em projetos de automação residencial, para controlar a iluminação, a temperatura, acesso à residência, cenas personalizadas de cômodos, irrigar plantas e hortas, entre outros. Saindo do âmbito residencial, é possível controlar dispositivos robóticos, tais como robôs móveis e manipuladores usando a voz, sem a necessidade de fios ou controles físicos (ROVAI, 2017).

Relata-se neste artigo o desenvolvimento de dois trabalhos de Iniciação Científica utilizando o tema Internet das Coisas, que tiveram como objetivo introduzir aos alunos os conceitos básicos e aplicações, envolvendo esta vasta área de pesquisa e desenvolvimento, que não fazem parte da maioria das grades curriculares dos cursos das escolas de engenharia.

\section{METODOLOGIA}

No primeiro projeto teve-se como objetivo aplicar os conceitos de IoT utilizando as assistentes de voz dos smartphones, o Google Assistant, para a automação de tarefas de uma residência, tais como acender/apagar uma lâmpada, abrir /fechar um portão. No segundo caso aplica-se a IoT para monitorar o ambiente (casa), utilizando sensores de temperatura, umidade, luminosidade e monóxido de carbono, e a partir destes o acionamento de lâmpadas e ventiladores, visando o conforto térmico e a segurança, respectivamente, usando para isso a internet sem fio e aplicativos. Com isso obtém-se um bom aprendizado sobre conceitos e dispositivos existentes no mercado envolvendo sensores, sistemas de comunicação sem fio, supervisão, e as interações próprias da Internet das Coisas. 
Para o desenvolvimento destes projetos foi necessário utilizar plataformas de sistemas embarcados abertas, como Arduino, e NodeMCU responsáveis por conectar os sensores/atuadores aos aplicativos e serviços (Middlewares).

\section{APLICATIVOS E SERVIÇOS}

Para se desenvolver sistemas IoT existem aplicativos e serviços (interfaces) visando controlar dispositivos conectados à internet por meio da voz. Além disso, são usados aplicativos de conexão e controladores para o interfaceamento entre o ser humano e o sistema IoT, tais como, os softwares, Blynk app, Google Assistant e IFFT (If This Than That ). Existem também os aplicativos que desenham gráficos para visualização de dados de sensores como o ThingSpeak, entre outros.

\subsection{Blynk app}

O Blynk app é um aplicativo feito pela Blynk, uma empresa que facilita o acesso e a conexão da internet das coisas em tempo real. Este aplicativo consegue se comunicar com a nuvem da própria empresa, via internet, e enviar informações para dispositivos IoT que estejam conectados à essa nuvem. Além de fácil utilização, o aplicativo se destaca quanto ao número de dispositivos IoT compatíveis, sendo possível utilizar outras plataformas de hardware (Raspberry Pi, Esp8266 e ESP32) para aplicar no mesmo projeto.

O Blynk app pode ser substituído por algumas outras aplicações, como o NodeRED, uma plataforma de programação feita também para IoT, porém de difícil instalação e conexão com o hardware, quando comparado ao Blynk. Pode-se também criar um aplicativo próprio para controlar o projeto, porém, com menos ações e conexões com dispositivos IoT e menor confiabilidade, restrito à aplicações de redes locais. Uma versão gratuita do Blynk app é disponibilizada para download, para isso deve ser criada uma conta individual, possibilitando a construção de protótipos (BLYNK INC, 2020).

\subsection{Google Assistant}

O Google Assistant é um serviço da empresa Google (GOOGLE, 2020), sendo uma assistente pessoal virtual, que pode realizar tarefas diárias para o usuário, chamadas, agendar eventos, mandar mensagens e abrir aplicativos. A assistente se preocupa em ter uma boa relação e responsividade com o usuário, pelo comando de "Ok Google" ou "Hey Google", dando respostas precisas e sucintas.

Outro fator importante desse serviço é sua ampla difusão, disponível em todos os smartphones com o sistema operacional Android (ANDROID, 2020), além de outros dispositivos "smart" como caixas de som, televisores, relógios e central multimídia veicular.

Com um poderoso software para o tratamento da voz, o serviço obtém resultados precisos, conforme as ações cotidianas, localização e rotina do usuário. Portanto, o usuário entra com o comando de voz pelo microfone do smartphone, que faz a captação e o tratamento do sinal de voz (treinamento) no aplicativo Google Assistant, transformando este comando em texto (código) (PÉREZ, 2017).

\subsection{Gateway}

A interface Gateway ou porta de ligação é uma máquina intermediária geralmente destinada a interligar redes, separar domínios de colisão, ou traduzir protocolos como os routers (ou roteadores) e firewalls, ambos servem de intermediários entre o usuário e a rede, para converter as informações entre redes heterogêneas, permitindo a comunicação entre 
diferentes ambientes e arquiteturas. Destaca-se que este tipo de sistema possui detecção de ataques para proteger a troca de informações entre a rede local e outras redes.

\subsection{IFFT}

O IFTTT ( If This Than That) é um serviço gratuito baseado na web que cria cadeias de instruções condicionais simples, chamadas de "Applets", sendo basicamente constituído por um gatilho, que dispara a ação, e uma consequência. Diversas empresas são parceiras do serviço, como a Google, Microsoft, GE, Spotify, Twitter e disponibilizam o acesso do serviço em seus produtos.

\subsection{A plataforma ThingSpeak}

O ThingSpeak é uma plataforma para implentar projetos em IoT que permite agregar, visualizar e analisar streams de dados, de uma forma muito simples, para envio de dados gerados no ESP32, Arduino ou qualquer outro dispositivo com recursos para comunicação em rede. Uma de suas grandes vantagens é a possibilidade de visualização dos dados enviados pelos dispositivos em tempo real, com a possibilidade de integração e análise pelo MatLab. É uma plataforma gratuita na web, sem a necessidade de aquisição de licença.

Possui várias ferramentas de análise, como visualização de dados em gráfico, e triggers para automatização de tarefas. O upload de dados se dá através de requisições HTTP/HTTPS contendo os dados, canais onde devem ser escritos e uma chave de autenticação de escrita do canal, caso este não seja público.

O ThingSpeak possui algumas limitações na versão gratuita, como o tempo entre upload de dados que deve ser de, no mínimo, 15 segundos, sob pena de serem ignorados ou não registrados e a quantidade de canais e widgets (atalhos aos apps) (THINGSPEAK, 2016).

\section{TRABALHOS DESENVOLVIDOS}

No primeiro projeto com o conceito de IoT utiliza-se o comando de voz de um smartphone para controlar dispositivos conectados a um controlador, onde ambos devem estar conectados à internet. No segundo projeto aplica-se o conceito de IoT para tornar uma casa inteligente usando sensores de temperatura, umidade, luminosidade e monóxido de carbono.

\subsection{Diagramas de blocos para a aplicação da IoT com o comando de voz.}

A proposta deste trabalho está baseada no Diagrama de blocos da Figura 1. Neste diagrama de blocos inicia-se o processo IoT utilizando o aplicativo Google Assistant e o comando de voz do usuário (pelo microfone do smartphone), que transforma este comando de voz em texto. O texto recebido à entrada do sistema é interpretado pelo Google Assistant, se o comando for reconhecido, ativa o protocolo IFTTT e acessa um link, correspondente ao servidor do próximo bloco, o Blynk. Para a configuração do serviço conecta-se a conta Google do usuário ao IFTTT, e cria-se um applet chamado "Ligar Relé". Escolhem-se os serviços Google Assistant e aplicativos necessários para serem ligados pelo IFTTT.

Por fim, pela IDE programa-se a placa Arduino Mega 2560, de forma a conectar-se à rede de internet cabeada do ambiente de laboratório, utilizando um endereço IP (Identifier Protocol) e um endereço MAC (Media Access Control), controle de acesso a placa de rede. Foi acoplado ao slot do Arduino para fazer a conexão com a rede local, o Ethernet Shield W5100 (CI Wiznet W5100). Após estas configurações, inicia-se a conexão com o servidor e os testes. 
Figura 1- Diagrama de blocos do primeiro projeto

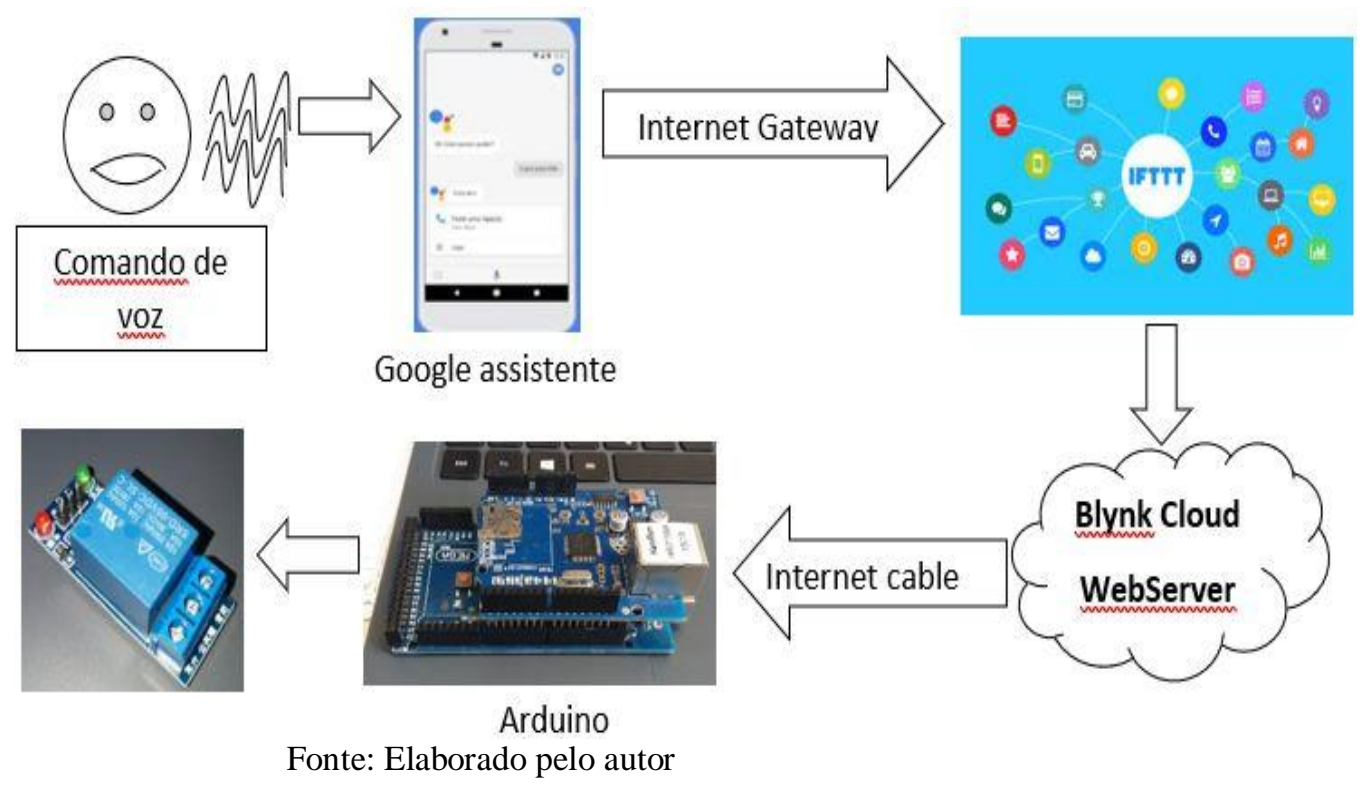

4.2 Diagramas de blocos do projeto da IoT para tornar uma casa inteligente.

Neste projeto teve-se como objetivo aplicar o conceito de IoT destinado a monitorar o ambiente de uma residência, por meio da utilização de alguns sensores, tais como a temperatura, umidade, luminosidade e monóxido de carbono, e por intermédio dessas informações fazer o acionamento de lâmpadas e ventiladores, visando o conforto térmico e a segurança no caso do monóxido de carbono, usando para isso a internet sem fio e aplicativos. O Diagrama de blocos geral para o sistema IoT implementado é apresentado na Figura 2.

Figura 2 - Diagrama de blocos geral loT para o segundo projeto.

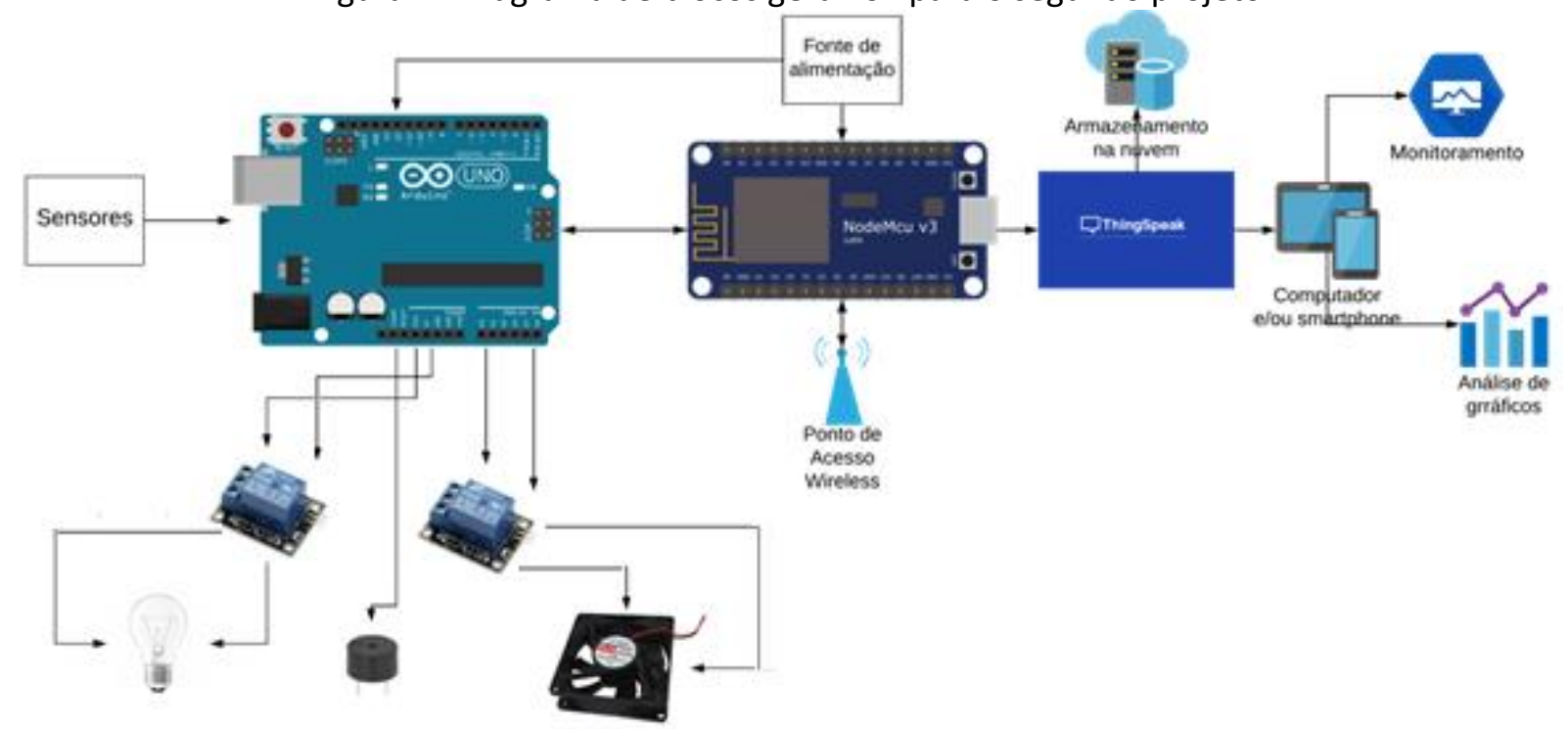

Fonte: Elaborado pelo autor

Neste diagrama inicia-se o processo a partir dos sensores que enviam os seus dados para o Arduino UNO R3, onde são armazenados os strings. Estas strings são enviadas ao 
NodeMCU através de uma comunicação serial realizada com o Arduino. Ambas as plataformas são alimentadas pela porta USB de um computador (IoT- GUIDER, 2019)

O NodeMcu conecta-se à internet por meio de um ponto de acesso e se comunica com a plataforma (middleware) ThingSpeak (2019), que pode ser acessado de qualquer lugar por computadores e/ou smartphones. Esta plataforma faz o monitoramento e pode disponibilizar o resultado da leitura dos sensores na forma gráfica, armazenando os resultados na nuvem.

O Arduino interpreta os dados dos sensores e pode decidir fazer o acionamento automático de uma lâmpada e/ou um ventilador por meio de relés, ou o buzzer caso da detecção de monóxido de carbono. O Arduino centraliza o controle do sistema e processa todos os comandos e acionamentos, fazendo o papel de servidor de aplicação.

\subsection{Conectando o Nodemcu ao Thinkspeak}

O ESP8266 NodeMcu faz a conexão à internet, por isso configura-se a IDE do Arduino para o uso do módulo. Para que fosse possível realizar o envio de dados à plataforma ThingSpeak foi necessário criar, previamente, uma conta gratuita no site. Ao criar uma conta no site deve-se criar um canal público (ou privado) onde são enviados os dados dos sensores, possibilitando a análise dos dados de forma gráfica e em tempo real. Neste projeto optou-se por um canal privado, visto que este seria usado em pesquisa acadêmica.

Realizou-se a conexão do NodeMcu com a internet através de um ponto de acesso em um computador com Windows 10 e com o servidor da ThingSpeak adicionando o nome e senha da rede wifi e a Api key à codificação fornecida pelo canal criado no site, assim como outros dados importantes, tais como, o campo de entrada de cada sensor e o IP do servidor.

\section{RESULTADOS}

Para um teste preliminar com o primeiro sistema IoT e a verificação do funcionamento do programa foram utilizados uma lâmpada e o módulo relé de um canal, mostrado na Figura 3, para o controle por comando de voz para acender/apagar a lâmpada.

Figura 3 - Conexão em funcionamento lâmpada.

(a) Acesa. (b) Apagada

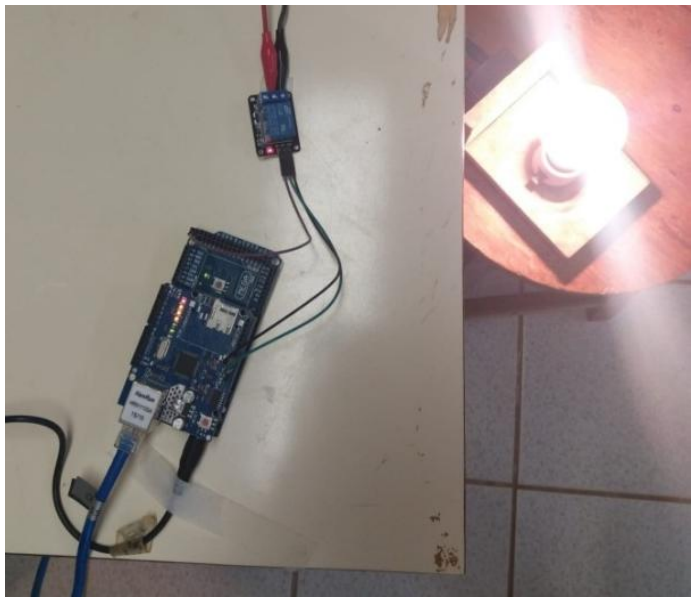

(a)

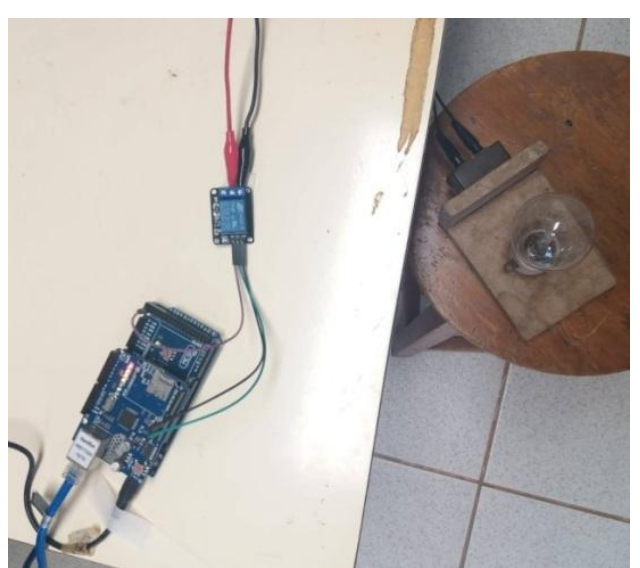

(b)

Fonte: Elaborado pelo autor

Apresentam-se a seguir, na Figura 4(a), os testes preliminares no protoboard e com o NodeMCU para o segundo projeto, utilizando os sensores MQ-7, DHT-11, sensor de gás e umidade e temperatura, respectivamente. Na Figura 4(b) mostram-se os testes para o sensor 
(C) COBENCE 2020

"Os desafios para formar hoje o engenheiro do amanhã"
O1 a 03 de dezembro Evento On-line

LDR (variação da resistência conforme a incidência de luz ambiente) que detecta a quantidade de luz ambiente tornando possível o monitoramento e a necessidade de acionar ou desligar as lâmpadas, visando economizar energia em uma casa. Para definir a faixa de luz detectada, nos testes foram utilizados o Arduino UNO R3 e 3 leds. Testou-se o sensor de gás MQ-7, simulando o vazamento de gás e o surgimento de fogo no ambiente, cujo alerta é dado por um buzzer com alimentação de $5 \mathrm{~V}$, que emite um sinal sonoro que permanecerá ativado até que o nível de gás volte ao normal.

Figura 1 - Teste com os dois sensores no ambiente.

(a) Com o NodeMcu. (b) Com o Arduino.

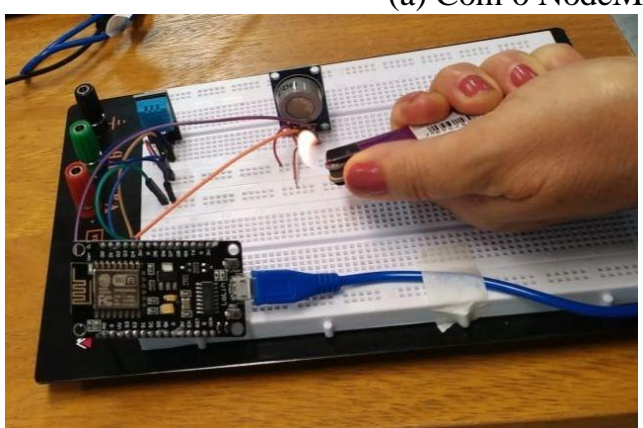

(a)

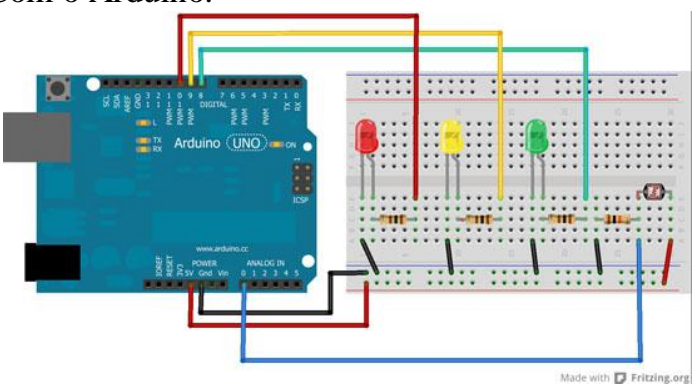

(b)

Fonte: Elaborado pelo autor.

Na Figura 5 (a) a (d) têm-se os resultados da variação de temperatura, umidade, monóxido de carbono e de sensor LDR que fornece a leitura da luminosidade do ambiente coletados pelo ThingSpeak na forma gráfica, respectivamente. O circuito completo do segundo projeto é mostrado na Figura 6.

Figura 5 - Resultados dos testes gerados no

ThingSpeak. (a) e (b) DHT11. (c) MQ -7. (d) LDR.

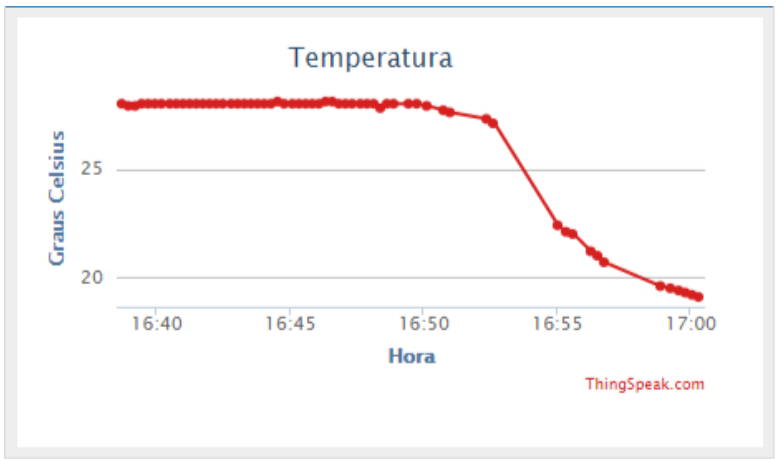

(a)

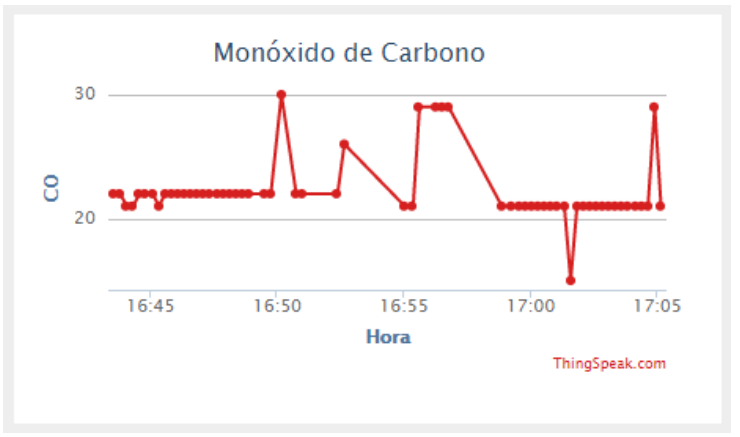

(c)

Fonte: Elaborado pelo autor

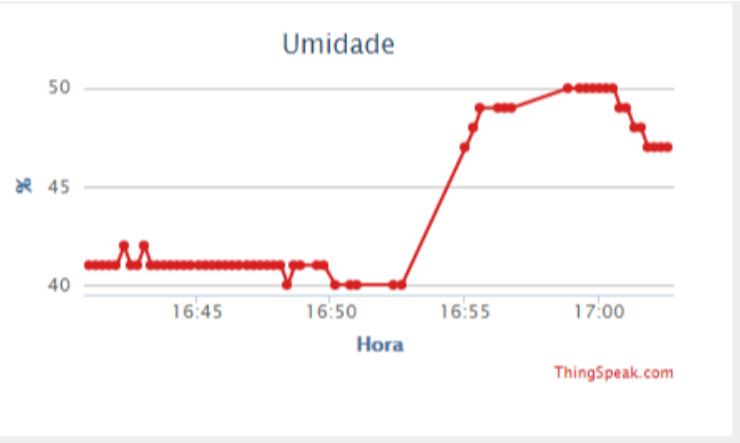

(b)

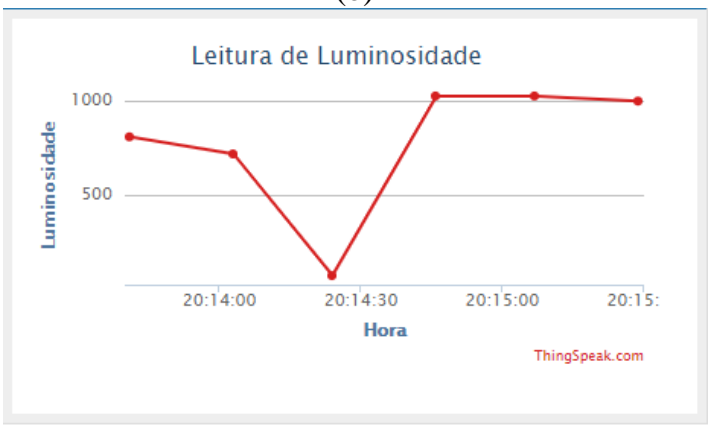

(d) 
No caso do sensor DHT-11 tem-se a leitura da temperatura em graus Celcius vs. hora, umidade em função do percentual relativo vs hora, para a leitura do monóxido do carbono em ppm vs hora e a luminosidade vs hora.

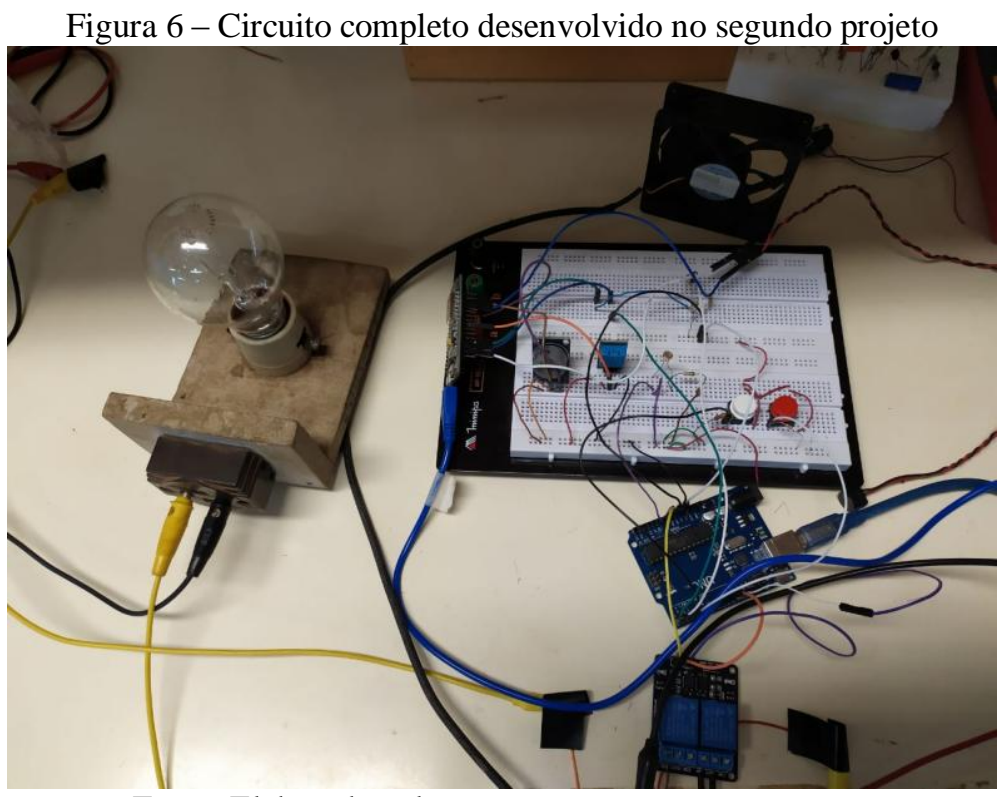

Fonte: Elaborado pelo autor

\section{CONSIDERAÇÕES FINAIS E CONTRIBUIÇÕES}

Por meio deste artigo foram apresentados dois trabalhos de pesquisa em Iniciação científica de alunos da graduação atraídos pelo tema da Internet das Coisas, pelos avanços científicos e tecnológicos que representa esta área de pesquisa atualmente, com a possibilidade do surgimento de novas ideias capazes de trazer mais conforto para a sociedade como um todo e por representar um mercado de trabalho emergente, ainda pouco explorado nos cursos acadêmicos da graduação em áreas afins como Engenharia Elétrica, Mecatrônica e de Ciência da Computação.

Como contribuição deste artigo, além da descrição dos projetos desenvolvidos pelos alunos, apresentam-se suas considerações a seguir, o que nos leva a concluir que estes projetos proporcionaram a eles uma melhor definição dos seus futuros acadêmico e profissional:

"A IC permitiu descobrir mais sobre a vida acadêmica; Interesse por novidades tecnológicas sobre o tema e a oportunidade de aprender mais, sobre a nova área; Aprendi muito sobre o tema IoT e aplicações; Mercado de trabalho emergente que eu gostaria de ter a oportunidade de trabalhar em uma empresa no futuro". A.B.C.T.

"A IC representou uma experiência no mundo acadêmico ao colocar em prática um projeto encontrado na internet atraído pelo tema IoT; O mundo de IoT é muito maior do que eu pensava; IoT se tornou minha área favorita; Tentar pesquisas mais elaboradas no tema IoT, mas em empresas". E.M.G

\section{REFERÊNCIAS}

ALEXANDRE, Gerônimo. B.; SILVA, Wesley M. A.; SILVA, Tiago E. Sistema de irrigação automático usando tecnologias de internet das coisas. In: Congresso Brasileiro 
"Os desafios para formar hoje o engenheiro do amanhã"

de Educação em Engenharia, 47, 2019, Fortaleza. Anais. Fortaleza: Abenge, 2019. Disponível em: http://www.abenge.org.br/cobenge/2019/anais.php. Acesso em: 07 nov. 2019.

ANDROID. Android. Disponível em: https://www.android.com/intl/pt-BR_br/. Acesso em: 06 fev. 2020.

BLYNK INC. Blynk. Disponível em: https://blynk.io/. Acesso em: 29 abr. 2020.

GOOGLE ASSISTANT. How to integrate Blynk and IFTTT. Disponível em: https://community.blynk.cc/t/how-to-integrate-blynk-and-ifttt-google-assistant/16107. Acesso em: 05 jun. 2019.

IOT-GUIDER. Serial Communication between NodeMCU and Arduino. 2018. Disponível em: <https://iot-guider.com/esp8266-nodemcu/serial-communication-between-nodemcu-andarduino/. Acesso em: 20 dez. 2019.

LIMA, Emanuel M. S.; NOBRE, A. Ygo Magalhães; ALENCAR de, Rômulo A. Ellery. Automação Residencial de Baixo Custo com Arduino Mega E Ethernet Shield. Trabalho de Conclusão de Curso. Centro Universitário Estácio do Ceará, 2016.

OLIVEIRA, Ricardo R. Uso do Microcontrolador Esp8266 para automação residencial. 2017. Trabalho de conclusão de curso (Graduação) - Escola Politécnica, Universidade Federal do Rio de Janeiro, [S. l.], 2017.

PÉREZ, Xose. Using Google Assistant to control your ESP8266 devices. 2017. Disponível em: <https://tinkerman.cat/post/using-google-assistant-control-your-esp8266-devices>. Acesso em: 05 jun. 2019.

ROCHA, Marcus V. Artigos em destaque das redes sociais às coisas conectadas, tudo no seu smartphone. 2019. Disponível em: <https://abinc.org.br/das-redes-sociais-as-coisasconectadas-tudo-no-seu-smartphone/>. Acesso em: 04 jun. 2019.

ROVAI, Marcelo. Controle ativado por voz com Android e NodeMCU. 2017. Disponível em: https://mjrobot.org/2017/03/29/controle-ativado-por-voz-com-android-e-nodemcu/. Acesso em: 04 jun. 2019.

SOARES, João L. L.; RODRIGO, M. dos Santos; BAIA, P. E. Costa; OLIVEIRA, G. S. Queiroz; NASCIMENTO, A. S. do. Domótica: Desenvolvimento de sistema de proteção para desligamento automático de válvula de fogão domiciliar com plataforma arduino®. In: CONGRESSO BRASILEIRO DE EDUCAÇÃO EM ENGENHARIA, 47, 2019, Fortaleza. Anais. Fortaleza: Abenge, 2019. Disponível em: http://www.abenge.org.br/cobenge/2019/anais.php. Acesso em: 07 nov. 2019.

SOUSA, Náiron de V. Automação Residencial por Comandos de Voz para Pessoas com Mobilidade Reduzida. 2018. 96 f. Dissertação (Mestrado) - Curso de Engenharia Elétrica, Instituto Federal da Paraíba, João Pessoa, 2018. Disponível em: http://repositorio.ifpb.edu.br/jspui/bitstream/177683/345/1/47\%20N\%C3\%A1 iron\%20de\%20 Vasconcelos\%20Sousa\%20\%20AUTOMA\%C3\%87\%C3\%83O\%20RESIDENCIAL\%20PO 
R\%20COMANDOS\%20DE\%20VOZ\%20PARA\%20PESSOAS\%20COM\%20MOBILIDAD E\%20REDUZIDA.pdf. Acesso em: 04 dez. 2019.

THINGSPEAK, Criar usuário. 2019. Disponível em: https://thingspeak.com/users/sign up. Acesso em: 25 jan. 2019.

\title{
SMALL PROJECTS INVOLVING INTERNET OF THINGS (IOT) ARE TOOLS THAT ENCOURAGE TO RESEARCH AND SCIENTIFIC INITIATION
}

\begin{abstract}
The internet is increasingly consolidated in people's daily lives as an essential tool by presenting new technologies and interactions, especially in times of pandemic. The Internet of Things (IoT) is a concept that was originated in the 90s, based on the connection of devices to the internet and, consequently, to man. It is a line of research in full development, that is why the interest of undergraduate students and the desire to undertake a scientific initiation on the topic is growing. In this article, two student undergraduate researches based on Internet of Things concept are presented, the first one uses the smartphone's voice assistant, Google Assistant, for the automation of tasks, such as turning on/off a lamp. It uses a cloud service called IFTTT (If This Than That) to connect the Google Assistant service and the Blynk App, to control an Arduino Mega 2560, with an ethernet shield and a relay that activate a circuit with a lamp. In the second one, the Internet of Things is approached to make a smart residential environment using the Arduino UNO R3 for the acquisition of data such as temperature, humidity, carbon monoxide and light sensors. It also controls the environment by reading these variables that are being monitored by using a NodeMCU as wifi connection to the ThingSpeak website server.
\end{abstract}

Keywords: Internet of things, Embedded systems, Sensors, Task automation. 